\title{
Weighted entropy of penta chains graph
}

\author{
Farkhanda Afzal $^{\mathrm{a}}{ }^{\odot}$ Mehmoona Abdul Razaq $^{\mathrm{b}}$ |Deeba Afzalb,* $^{\circ}$ |Saira Hameedc $^{\mathrm{s}}$
}

aDepartment of Humanities and Basic Sciences, Military College of Signals, National University of Sciences and Technology, 44000, Islamabad, Pakistan

${ }^{b}$ Department of Mathematics and Statistics, The University of Lahore, 54000, Lahore, Pakistan

cDepartment of Mathematics, University of the Punjab, 54000, Lahore, Pakistan
Mathematical chemistry is a branch of theoretical chemistry in which we predict the mathematical structure by means of mathematical tools. In past few decades, many studies have been conducted in this area. This theory has cooperated a significant role in the field of chemistry. The main goal of this study is to calculate the weighted entropies of penta chains. We studied the graph entropies with Randić index, Zagreb indices, atom-bond connectivity, augmented Zagreb index, geometric arithmetic index, and sum connectivity index. We obtained the weighted entropies for the graphs formed of concatenated 5cycles in one rows and in two rows of various lengths.

\section{KEYWORDS}

Molecular graph; topological indices; weighted entropy; penta chains.

Deeba Afzal

Email:deebafzal@gmail.com

Tel.: +92 3351572532

\section{Introduction}

For the QSAR research, the most practical option is the topological index. The Weiner index is used for establishing interrelationship model between the different chemical substances. In 1995 Yang and Yen evaluated a general expression of polycyclic graphs with unequal length for Weiner indices [1]. The Weiner index for the penta chain in two rows of unequal lengths was computed by A. laxmi and N. Prabhakar Rao [7]. In the last 50 years, the investigations into the information content of graphs and networks have been based on the profound and initial works of Shannon [2] and [3]. In order to measure the structural complexity of graphs and networks, the concept of graph entropy has been proposed [4,8]. Determining the complexity of the graphs has been used in various led of sciences, including information theory, biology, chemistry, and sociology.

There are different applications of graph entropy in communications and economics. We used the concept of graph entropy as a weighted graph, as in [6] who solved the problem of weighted chemical graph entropy by using special information functional. Some degree-based indices are characterized by investigating the extremes of the entropy of certain class of molecular graphs [5,9]. In this work, we computed the graph entropy for concatenated 5-cycles in one rows and in two rows of various lengths by taking Randić index, Zagreb indices, atom-bond connectivity, augmented Zagreb index, geometric arithmetic index and sum connectivity index. Some important formulas of degree based topological indices are presented in Table 1. 
TABLE 1 Degree based topological indices

\begin{tabular}{ccc}
\hline Topological Index & Notation & Formula \\
\hline Randic & $\mathrm{R}(\mathrm{G})$ & $\sum_{g h \in E(G)} \frac{1}{\sqrt{d_{g} \cdot d_{h}}}$ \\
Reciprocal Randic & $\mathrm{RR}(\mathrm{G})$ & $\sum_{g h \in E(G)} \sqrt{d_{g} \cdot d_{h}}$ \\
Frist Zagreb Second & $\mathrm{M}_{1}(\mathrm{G}) \mathrm{M}_{2}(\mathrm{G})$ & $\sum_{g h \in E(G)}\left(d_{g}+d_{h}\right)$ \\
Zagreb & $\mathrm{N}$ & $\sum_{g h \in E(G)}\left(d_{g} \cdot d_{h}\right)$ \\
Atom-bond connectivity & $\mathrm{ABC}(\mathrm{G})$ & $\sum_{g h \in E(G)} \sqrt{\frac{d_{g}+d_{h}-2}{d_{g} \cdot d_{h}}}$ \\
Agumented Zagred & $\mathrm{AZI}(\mathrm{G})$ & $\sum_{g h \in E(G)}\left(\frac{d_{g} \cdot d_{h}}{d_{g}+d_{h}-2}\right)^{3}$ \\
Geometric arithmetic & $\mathrm{GA}(\mathrm{G})$ & $\sum_{g h \in E(G)} \frac{2 \sqrt{d_{g} \cdot d_{h}}}{d_{g}+d_{h}}$ \\
Harmonic index & $\mathrm{HI}(\mathrm{G})$ & $\sum_{g h \in E(G)} \frac{2}{d_{g}+d_{h}}$ \\
Sum connectivityindex & $\mathrm{SCI}(\mathrm{G})$ & $\sum_{g h \in E(G)} \frac{1}{\sqrt{d_{g}+d_{h}}}$ \\
\hline
\end{tabular}

\section{Entropy}

The entropy of a graph is a functional depending both on the graph itself and on a probability distribution on its vertex set. This graph functional originated from the problem of source coding in information theory and was introduced by J. Krner in 1973. Although the notion of graph entropy has its roots in information theory, it was proved to be closely related to some classical and frequently studied graph theoretic concepts. For example, it provides an equivalent definition for a graph to be perfect and it can also be applied to obtain lower bounds in graph covering problems.

Definition 1.1. (Entropy). Let the probability density function

$$
P_{i j}=\frac{w(u v)}{\sum W(u v)}
$$

Then the entropy of graph $G$ is defined as

$$
I(G, w)=\sum P_{i j} \log P_{i j} \text {. }
$$

\section{Entropy of straight chaining of pentagons}

A straight chaining is a graph consisting of $n$ pentagonal cycle, each of two consecutive cycles have a common edge, making a chain denoted by $G(n, S)$. The order of $G(n, S)$ is $3 n+2$ and size $4 n+1$, and the diameter is $n+2$, $\mathrm{n} \geq 2$. (Figure 1 )

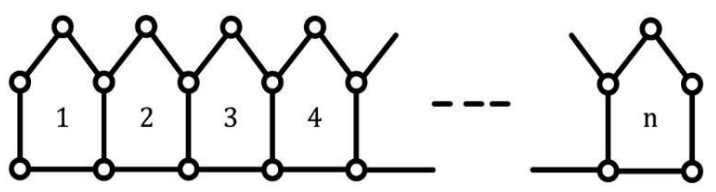

FIGURE 1 Straight chaining of pentagons $\mathrm{G}(\mathrm{n} ; \mathrm{S})$

TABLE 2 Edge partition of straight chaining of pentagons

\begin{tabular}{|c|c|c|c|c|}
\hline Edge & $\left(d_{G}(g), d_{G}(h)\right)$ & $(2,2)$ & $(2,3)$ & $(3,3)$ \\
\hline Vertices & Frequencies & 4 & $2 \mathrm{n}$ & $2 \mathrm{n}-3$ \\
\hline
\end{tabular}

Theorem 2.1. For the straight chain of pentagons. The weighted entropy with Randić index weight is: 
$I(G, R)=\log (1.4831632476 n+1)+0.5525899692$

$+\frac{0.0842380208}{n}+0.63575975 n$.

Proof. Using Table 2 values in the definition of Randić index we have

$$
\begin{aligned}
& R(G(n, S))=1.48316324276 n+1 I(G, R(n, S)) \\
& =\log (R)-\frac{1}{R} \sum_{g h \in E(G)}\left(\frac{1}{\sqrt{d_{g} \cdot d_{h}}} \log \frac{1}{\sqrt{d_{g} \cdot d_{h}}}\right) \\
& =\log (1.48316324276 n+1)-\frac{1}{1.4831632476 n+1} \\
& \left(4 .\left(\frac{1}{\sqrt{2.2}} \log \frac{1}{\sqrt{2.2}}\right)+2 n \cdot\left(\frac{1}{\sqrt{2.3}} \log \frac{1}{\sqrt{2.3}}\right)\right. \\
& \left.+(2 n-3) \cdot\left(\frac{1}{\sqrt{3.3}} \log \frac{1}{\sqrt{3.3}}\right)\right) \\
& =\log (1.48316324276 n+1)-\left(1+\frac{0.6742346142}{n}\right) \\
& (-0.6020599913-0.3176789177 n-0.3180808365 n \\
& +0.4771212548)=\log (1.48316324276 n+1) \\
& -(-0.6357597542 n-0.1249387365 \\
& \left.-0.4286512326-\frac{0.0842380208}{n}\right) \\
& =\log (1.48316324276 n+1)+0.5535899692 \\
& +\frac{0.0842380208}{n}+0.6357597542 n .
\end{aligned}
$$

Theorem 2.2. For the straight chain of pentagons. The weighted entropy with reciprocal Randić index weight is $I(G, R R)=\log (10.8989794856 n-1)-2.3233969748$ $+\frac{0.1730300832}{n}+4.7688010345 n$.

Proof. Using Table 2 values in the definition of reciprocal Randić index we have $R R(G(n, S))=10.8989794856 n-1 I(G, R R(n, s))$ $=\log (R R)-\frac{1}{R R} \sum_{g h \in E(G)}\left(\sqrt{d_{g} \cdot d_{h}} \sqrt{d_{g} \cdot d_{h}}\right)$ $=\log (10.8989794856 n-1)-\frac{1}{10.8989794856 n-1}$ $(4 . \sqrt{2.2} \log \sqrt{2.2}+2 n \cdot \sqrt{2.3} \log \sqrt{2.3}+(2 n-3) \sqrt{3.3} \log \sqrt{3.3})$ $=\log (10.8989794856 n-1)-\left(\frac{0.0917517095}{n}-1\right)$ $(2.4082399653+1.9060735062 n$ $+2.8627275283 n-4.2940912925)$

$=\log (10.8989794856 n-1)-\left(\frac{-0.1730300832}{n}\right.$ $+0.4375456472+1.8858513276-4.7688010345 n)$ $=\log (10.8989794856 n-1)-2.3233969748$ $+\frac{0.1730300832}{n}+4.7688010345 n$.
Theorem 2.3. For the straight chain of pentagons. The weighted entropy with Zagreb indices weight is

$$
I\left(G, M_{1}\right)=\log (22 n-2)-2.9290410986
$$

(a) $+\frac{0.198807393}{n}+8.163757524 n$

$$
I\left(G, M_{2}\right)=\log (30 n-11)-2.3503139951
$$

(b) $+\frac{0.5377195963}{n}+2.4103800159 n$.

Proof. (a) Using Table 2 values in the definition of Zagreb indices we have $M_{1}(G(n, S))=22 n-2 I\left(G, M_{1}\right)$

$=\log \left(M_{1}\right)-\frac{1}{M_{1}} \sum_{g h \in E(G)}\left(\left(d_{g}+d_{h}\right) \log \left(d_{g}+d_{h}\right)\right)$

$=\log (22 n-2)-\frac{1}{22 n-2}(4.4 \log 4+2 n .5 \log 5$

$+(2 n-3) .6 \log 6)=\log (22 n-2)-(0.0454545455 n-0.5)$

$(9.6329598612+6.9897000434 n+9.3378150046 n$

$-14.0067225069)$

$=\log (22 n-2)-\left(\frac{-0.1988073932}{n}+0.7421597757\right.$

$+2.1868813229-8.1637575254 n)$

$=\log (22 n-2)-2.9290410986+\frac{0.198807393}{n}$

$+8.163757524 n$

$M_{2}(n, S)=30 n-11 I\left(G, M_{2}\right)$

$=\log \left(M_{2}\right)-\frac{1}{M_{2}} \sum_{g h \in E(G)}\left(d_{g} \cdot d_{h}\right) \log \left(d_{g} \cdot d_{h}\right)$

$=\log (30 n-11)-\frac{1}{30 n-11}$ (4.(2.2) $\log (2.2)$

$+2 n .(2 \cdot 3 \log (2 \cdot 3))+(2 n-3) .(3 \cdot 3) \log (3 \cdot 3))$

$=\log (30 n-11)-\left(\frac{0.0333333333}{n}-0.0909090909\right)$

$(9.6329598612+9.3378150046 n+17.1763651699 n$ $-25.7645477549)$

$=\log (30 n-11)-\left(0.8838060049-\frac{0.5377195965}{n}\right.$

$-2.4103800159 n+1.4665079902)$

$=\log (30 n-11)-2.3503139951$

$+\frac{0.5377195963}{n}+2.4103800159 n$.

Theorem 2.4. For the straight chain of pentagone the weighted entropy with atombond connectivity index weight is $I(G, A B C)=\log (2.7475468957 n+0.8284271247)$ $+0.2516951221+\frac{0.0267650334}{n}+0.5403597774 n$. Proof. Using Table 2 values in the definition of atom-bond connectivity index we have 
$A B C(G(n, S))=2.7475468957 n+0.8284271247 I(G, A B C)$

$=\log (A B C)-\frac{1}{A B C} \sum_{g h \in E(G)}\left(\sqrt{\frac{d_{g}+d_{h}-2}{d_{g} \cdot d_{h}}} \log \sqrt{\frac{d_{g}+d_{h}-2}{d_{g} \cdot d_{h}}}\right)$

$=\log (2.7475468957 n+0.8284271247)$

$-\frac{1}{2.7475468957 n+0.8284271247}\left(4 \cdot \sqrt{\frac{2+2-2}{2.2}}\right.$

$\log \sqrt{\frac{2+2-2}{2.2}}+2 n \cdot \sqrt{\frac{2+3-2}{2.3}} \log \sqrt{\frac{2+3-2}{2.3}}$

$\left.+(2 n-3) \cdot \sqrt{\frac{3+3-2}{3+3}} \log \sqrt{\frac{3+3-2}{3.3}}\right)$

$=\log (2.7475468957 n+0.8284271247)-\left(\frac{0.3639610307}{n}\right.$

$+1.20710667813)(-0.4257207025-0.2128603513 n$

$-0.2347883454 n+0.3521825181)$

$=\log (2.7475468957 n+0.8284271247)-\left(\frac{-0.0267650334}{n}\right.$

$-0.162926681-0.0887684411-0.5403597774 n)$

$=\log (2.7475468957 n+0.8284271247)+0.2516951221$

$+\frac{0.0267650334}{n}+0.5403597774 n$.

Theorem 2.5. For the straight chain of pentagone the weighted entropy with augmented Zagreb index weight is $I(G, A Z I)=\log (92.125 n-104.6875)+0.1058993658$ $-\frac{0.3050905715}{n}+0.1430706968 n$.

Proof. Using Table 2 values in the definition of augmented Zagreb index we have $A Z I(G(n, S))=92.125 n-104.6875 I(G, A Z I)$

$=\log (A Z I)-\frac{1}{A Z I} \sum_{g h \in E(G)}\left(\left(\frac{d_{g} \cdot d_{h}}{d_{g}+d_{h}-2}\right)^{3} \log \left(\frac{d_{g} \cdot d_{h}}{d_{g}+d_{h}-2}\right)^{3}\right)$

$=\log (92.125 n-104.6875)-\frac{1}{92.125 n-104.6875}$

(4. $\left(\frac{2.2}{2+2-2}\right)^{3} \log \left(\frac{2.2}{2+2-2}\right)^{3}+2 n \cdot\left(\frac{2.3}{2+3-2}\right)^{3}$

$\left.\times \log \left(\frac{2.3}{2+3-2}\right)^{3}+(2 n-3) \cdot\left(\frac{3.3}{3+3-2}\right)^{3} \log \left(\frac{3.3}{3+3-2}\right)^{3}\right)$

$=\log (92.125 n-104.6875)-\left(\frac{0.0108548168}{n}\right.$

$-0.0095522388)(28.8988795837+14.4494397919 n$

$+0.5282737772 n-0.7924106658)=\log (92.125 n-104.6875)$

$-\left(\frac{0.3050905717}{n}+0.1625803372-0.2684797031\right.$

$-0.1430706968 n)=\log (92.125 n-104.6875)$

$+0.1058993658-\frac{0.3050905715}{n}+0.1430706968 n$.

Theorem 2.6. For the straight chain of pentagone the weighted entropy with geometric-arithmetic index weight is $I(G, G A)=\log (13.9191835885 n+1)$ $+0.0043869606+0.01737057313 n$.
Proof. Using Table 2 values in the definition of geometric-arithmetic Index we have $G A(G(n, S))=3.9595917942 n+1 I(G, G A)$

$=\log (G A)-\frac{1}{G A} \sum_{g h \in E(G)}\left(\frac{2 \sqrt{d g \cdot d h}}{d_{g}+d_{h}} \log \frac{2 \sqrt{d_{g} \cdot d_{h}}}{d_{g}+d_{h}}\right)$

$=\log (3.9191835885 n+1)-\frac{1}{3.9191835885 n+1}$

(4. $\frac{2 \sqrt{2.2}}{2+2} \log \frac{2 \sqrt{2.2}}{2+2}+2 n \cdot \frac{2 \sqrt{2.3}}{2+3} \log \frac{2 \sqrt{2.3}}{2+3}$

$\left.+(2 n-3) \cdot \frac{2 \sqrt{3.3}}{3+3} \log \frac{2 \sqrt{3.3}}{3+3}\right)$

$=\log (3.9191835885 n+1)-\left(\frac{0.2525512861}{n}+1\right)$

$(-0.0173705731 n)=\log (3.9191835885 n+1)$

$+0.0043869606+0.01737057313 n$.

Theorem 2.7. For the straight chain of pentagone the weighted entropy with harmonic index weight is

$I(G, H)=\log (1.4666666667 n+1)+0.5588702206$

$+\frac{0.0851855022}{n}+0.364328434 n$.

Proof. Using Table 2 values in the definition of harmonic index we have

$H(G(n, S))=1.4666666667 n+1 I(G, H)$

$=\log (H I)-\frac{1}{H I} \sum_{g h \in E(G)}\left(\frac{2}{d_{g}+d_{h}} \log \frac{2}{d_{g}+d_{h}}\right)$

$=\log (1.4666666667 n+1)-\frac{1}{1.4666666667 n+1}$

(4. $\left.\frac{2}{2+2} \log \frac{2}{2+2}+2 n \cdot \frac{2}{2+3} \log \frac{2}{2+3}+(2 n-3) \cdot \frac{2}{3+3} \log \frac{2}{3+3}\right)$

$=\log (1.4666666667 n+1)-\left(\frac{0.6818181818}{n}+1\right)$

$(-0.6020599913-0.3183520069 n$

$-0.3180808365 n+0.4771212548)$

$=\log (1.4666666667 n+1)-\left(-\frac{0.0851855022}{n}\right.$

$-0.4339314841-0.1249387365-0.6364328434 n)$

$=\log (1.4666666667 n+1)+0.5588702206$

$+\frac{0.0851855022}{n}+0.6364328434 n$.

Theorem 2.8. For the straight chain of pentagone the weighted entropy with sum connectivity index weight is

$I(G, S C I)=\log (1.710923772 n+0.775255128)$

$+0.5303145315+\frac{0.0733765097}{n}+0.8129811507 n$. 


\section{Proof.}

$S C I(G(n, S))=1.7109237719 n+0.7752551286 I(G, S C I)$

$=\log (S C I)-\frac{1}{S C I} \sum_{g h \in E(G)}\left(\frac{1}{\sqrt{d_{g}+d_{h}}} \log \frac{1}{\sqrt{d_{g}+d_{h}}}\right)$

$=\log (1.710923772 n+0.775255128)$

$-\frac{1}{1.710923772 n+0.775255128}\left(4 \cdot \frac{1}{\sqrt{2+2}} \log \frac{1}{\sqrt{2+2}}\right.$

$\left.+2 n \cdot \frac{1}{\sqrt{2+3}} \log \frac{1}{\sqrt{2+3}}+(2 n-3) \cdot \frac{1}{\sqrt{3+3}} \log \frac{1}{\sqrt{3+3}}\right)$

$=\log (1.710923772 n+0.775255128)-\left(\frac{0.5844795756}{n}\right.$

$+1.2898979486)(-0.1255416148-0.6302678065 n)$

$=\log (1.710923772 n+0.775255128)+0.5303145315$

$+\frac{0.0733765097}{n}+0.8129811507 n$.

\section{Double row pentachains $G\left(n ; S_{1}\right)$}

In this section, we obtain the entropies of graphs consisting of two rows of straight chains with $\mathrm{n}$ pentagons in two rows combined, as shown in Figure 2.

It can be observed from Figure 2 that there are following four types of edges.

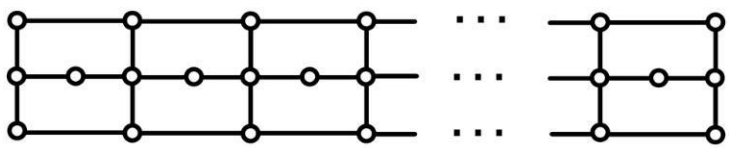

FIGURE 2 Double row pentachains $\mathrm{G}\left(\mathrm{n} ; \mathrm{S}_{1}\right)$

TABLE 3 Edge partition of Double row pentachains $\mathrm{G}\left(\mathrm{n}, \mathrm{S}_{1}\right)$

\begin{tabular}{|c|c|c|c|c|c|}
\hline Edge & $\left(d_{G}(g), d_{G}(h)\right)$ & $(2,2)$ & $(2,4)$ & $(3,3)$ & $(3,4)$ \\
\hline Vertices & Frequencies & 10 & $2 \mathrm{n}-2$ & $2 \mathrm{n}-4$ & $2 \mathrm{n}-2$ \\
\hline
\end{tabular}

Theorem 3.1. For the double row pentachains $\mathrm{G}\left(\mathrm{n} ; \mathrm{S}_{1}\right)$ the weighted entropy with Randić index weight is

$I(G, R)=\log (1.951123717 n+1.4646925209)$

$+0.7061549932+\frac{0.1650130582}{n}+0.6478562165 n$.

Proof. Using table 3 values in the definition of Randić index we have

$R\left(G\left(n, S_{1}\right)\right)=1.951123717 n+1.4646925209 I(G, R)$

$=\log (R)-\frac{1}{R} \sum_{g h \in E(G)}\left(\frac{1}{\sqrt{d_{g} \cdot d_{h}}} \log \frac{1}{\sqrt{d_{g} \cdot d_{h}}}\right)$

$=\log (1.951123717 n+1.4646925209)$

$-\frac{1}{1.951123717 n+1.4646925209}\left(10 \cdot \frac{1}{\sqrt{6}} \log \frac{1}{\sqrt{6}}\right.$

$+(2 n-2) \times \frac{1}{\sqrt{8}} \log \frac{1}{\sqrt{8}}+(2 n-4) \times \frac{1}{\sqrt{9}} \log \frac{1}{\sqrt{9}}$

$\left.+(2 n-2) \times \frac{1}{\sqrt{12}} \log \frac{1}{\sqrt{12}}\right)$ $=\log (1.951123717 n+1.4646925209)$

$-\left(\frac{0.5125251624}{n}+0.6827371518\right)(-1.5883945883$

$-0.3192965269 n+0.3192905269-0.3180808365 n$

$+0.636161673-0.3115327915 n+0.3115327915)$

$=\log (1.951123717 n+1.4646925209)$

$-\left(-\frac{0.1650130582}{n}-0.4863403312\right.$

$-0.219814662-0.6478562165 n)$

$=\log (1.951123717 n+1.4646925209)+0.7061549932$

$+\frac{0.1650130582}{n}+0.6478562165 n$.

Theorem 3.2. For the double row pentachains $G\left(n ; S_{1}\right)$ the weighted entropy with reciprocal Randc index weight is

Proof. Using Table 3 values in the definition of reciprocal Randić index we have 
$\left.R R\left(G\left(n, S_{1}\right)\right)=18.5850514798 n-0.0901600519 I(G, R R)\right)$

$=\log (R R)-\frac{1}{R R} \sum_{g h \in E(G)}\left(\sqrt{d_{g} \cdot d_{h}} \log \sqrt{d_{g} \cdot d_{h}}\right)$

$=\log (18.5850514798 n-0.0901600519)-\left(\frac{0.0538066671}{n}\right.$

$-11.0913866943)(9.5303675298-2.5543242152$

$-5.7254550566-3.7383934974+2.5543242153 n$

$+2.8627275283 n+3.7383934974 n)$

$=\log (18.5850514798 n-0.0901600519)-(0.4926239938$

$\left.-\frac{0.1338605082}{n}-101.5465835263 n+27.5932099203\right)$

$=\log (18.5850514798 n-0.0901600519)-28.0858339141$

$+\frac{0.1338605082}{n}+101.5465835263 n$.

Theorem 3.3. For the double row pentachains $\mathrm{G}(\mathrm{n} ; \mathrm{S} 1)$ the weighted entropy with Zagreb indices weight is

$[a] I\left(G, M_{1}\right)=\log (38 n)-0.8028158579+\frac{1.3598997417}{n}$

$[b] I\left(G, M_{2}\right)=\log (58 n-16)-2.7426710691$

$+\frac{0.482990431}{n}+3.5953846792 n$.

Proof. Using Table 3 values in the definition of Zagreb Indices we have

$M_{1}\left(G\left(n, S_{1}\right)\right)=38 n I\left(G, M_{1}\right)$

$=\log \left(M_{1}\right)-\frac{1}{M_{1}} \sum_{g h \in E(G)}\left(\left(d_{g}+d_{h}\right) \log \left(d_{g}+d_{h}\right)\right)$

$=\log (38 n)-\left(\frac{0.0263157895}{n}\right)(34.9485002168$

$+(2 n-2)(4.6689075023)+(2 n-4)(4.6689075023)$

$+(2 n-2)(5.9156862801))=\log (38 n)-\left(\frac{0.0263157895}{n}\right)$

$(30.5070025694 n-51.6761901342)$

$=\log (38 n)-0.8028158579+\frac{1.3598997417}{n}$

$M_{2}\left(G\left(n, S_{1}\right)\right)=58 n-16 I\left(G, M_{2}\right)$

$=\log \left(M_{2}\right)-\frac{1}{M_{2}} \sum_{g h \in E}\left(\left(d_{g} \cdot d_{h}\right) \log \left(d_{g} \cdot d_{h}\right)\right)$

$=\log (58 n-16)-\left(\frac{0.0172413793}{n}-0.0625\right)$

(46.689075023-14.4494397919-25.9003499052

$-34.35273034+25.9003499052 n$

$+14.4494397919 n+17.1763651699 n)$

$=\log (58 n-16)-\left(0.9918302557-\frac{0.482990431}{n}\right.$

$+1.7508403134-3.5953846792 n)$

$=\log (58 n-16)-2.7426710691$

$+\frac{0.482990431}{n}+3.5953846792 n$.
Theorem 3.4. For the double row pentachains $\mathrm{G}(\mathrm{n} ; \mathrm{S} 1)$ the weighted entropy with atom-bond connectivity index weight is

$I(G, A B C)=\log (4.338201173 n+1.099873477)$

$-1.5069922084-\frac{0.4172566367}{n}+0.547393474 n$.

Proof. Using Table 3 values in the definition of atom-bond connectivity Index we have

$A B C\left(G\left(n, S_{1}\right)\right)=4.338201173 n+1.099873477 I(G, A B C)$

$=\log (A B C)-\frac{1}{A B C} \sum_{g h \in E}\left(\sqrt{\frac{d_{g}+d_{h}-2}{d_{g} \cdot d_{h}}} \log \sqrt{\frac{d_{g}+d_{h}-2}{d_{g} \cdot d_{h}}}\right)$

$=\log (4.338201173 n+1.099873477)$

$-\frac{1}{4.338201173 n+1.099873477}\left(10 \cdot \sqrt{\frac{2+3-2}{2.3}} \log \sqrt{\frac{2+3-2}{2.3}}\right.$

$+(2 n-2) \sqrt{\frac{2+4-2}{2.4}} \log \sqrt{\frac{2+4-2}{2.4}}+(2 n-4) \sqrt{\frac{3+3-2}{3+3}}$

$\left.\log \sqrt{\frac{3+3-2}{3.3}}+(2 n-2) \sqrt{\frac{3+4-2}{3+4}} \log \sqrt{\frac{3+4-2}{3.4}}\right)$

$=\log (4.338201173 n+1.099873477)$

$-\frac{1}{4.338201173 n+1.099873477}(-1.0643017563$

$+0.2128603513+0.2875558219+0.2454253012$

$-0.2128603513 n-0.143777911 n-0.2454253012 n$ )

$=\log (4.338201173 n+1.099873477)-\left(\frac{0.4172566367}{n}\right.$

$-0.1387818452+1.6457740536-0.547393474 n)$

$=\log (4.338201173 n+1.099873477)-1.5069922084$

$-\frac{0.4172566367}{n}+0.547393474$.

Theorem 3.5. For the double row pentachains $\mathrm{G}(\mathrm{n} ; \mathrm{S} 1)$ the weighted entropy with augmented Zagreb index weight is $I(G, A Z I)=\log (66.42925 n-9.2105)-3.429853578$ $+\frac{0.3293342923}{n}+7.606009991 n$.

Proof. Using Table 3 values in the definition of augmented Zagreb Index we have 
$A Z I\left(G\left(n, S_{1}\right)\right)=66.42925 n-9.2105 I(G, A Z I)$

$=\log (A Z I)-\frac{1}{A Z I} \sum_{g h \in E(G)}\left(\left(\frac{d_{g} \cdot d_{h}}{d_{g}+d_{h}-2}\right)^{3} \log \left(\frac{d_{g} \cdot d_{h}}{d_{g}+d_{h}-2}\right)^{3}\right)$

$=\log (66.42925 n-9.2105)-\frac{1}{66.42925 n-9.2105}(10.8 \log 8$

$+(2 n-2) 8 \log 8+(2 n-4)\left(\frac{9}{4}\right)^{3} \log \left(\frac{9}{4}\right)^{3}+(2 n-2)(13.824)$

$\log (13.824))=\log (66.42925 n-9.2105)-\left(\frac{0.0150536097}{n}\right.$

$-0.1085717388)(72.24719896-14.44943979$

$-48.13894796-31.53624124$

$+14.44943979 n+24.06947398 n+31.53624124 n)$

$=\log (66.42925 n-9.2105)-\left(\frac{0.3293342923}{n}+1.054582959\right.$

$+2.375270619-7.606009991 n)=\log (66.42925 n-9.2105)$

$-3.429853578+\frac{0.3293342923}{n}+7.606009991 n$.

Theorem 3.6. For the double row pentachains $\mathrm{G}\left(\mathrm{n} ; \mathrm{S}_{1}\right)$ the weighted entropy with geometric-arithmetic Zagreb index weight is

$I(G, G A)=\log (5.8651047204 n+1.9328542508)$ $+0.0251323338+\frac{0.2491133952}{n}+0.02953699262 n$.

Proof. Using Table 3 values in the definition of Geometric-Arithmetic Index we have $G A\left(G\left(n, S_{1}\right)\right)=5.8651047204 n+1.9328542508 I(G, G A)$ $=\log (G A)-\frac{1}{G A} \sum_{g h \in E(G)}\left(\frac{2 \sqrt{d_{g} \cdot g_{h}}}{d_{g}+d_{h}} \log \frac{2 \sqrt{d_{g} \cdot g_{h}}}{d_{g}+d_{h}}\right)$ $=\log (5.8651047204 n+1.9328542508)-\left(\frac{0.1704999395}{n}\right.$ $+05173695841)(-0.0868528657+0.04822706066$ $+0.008862995-0.00862995 n-0.048227706066 n)$ $=\log (5.8651047204 n+1.9328542508)-(-0.009733961$ $\left.-\frac{0.00507455574}{n}-0.02953699262 n-0.0153983728\right)$ $=\log (5.8651047204 n+1.9328542508)+0.0251323338$ $+\frac{0.2491133952}{n}+0.02953699262 n$.

Theorem 3.7. For the double row pentachains $\mathrm{G}\left(\mathrm{n} ; \mathrm{S}_{1}\right)$ the weighted entropy with harmonic Zagreb index weight is $I(G, H I)=\log (1.904761905 n+1.428571429)$ $+0.73007460609+\frac{0.171476281}{n}+0.6685853473 n$.

Proof. Using Table 3 values in the definition of Harmonic index we have
$H I\left(G\left(n, S_{1}\right)\right)=1.904761905 n+1.428571429 I(G, H I)$

$=\log (H I)-\frac{1}{H I} \sum_{g h \in E(G)} \frac{2}{d_{g}+d_{h}} \log \frac{2}{d_{g}+d_{h}}$

$=\log (1.904761905 n+1.428571429)$

$-\frac{1}{1.904761905 n+1.428571429}\left(10 \frac{2}{5} \log \frac{2}{5}\right.$

$\left.+(2 n-2) \frac{2}{6} \log \frac{2}{6}+(2 n-4) \frac{2}{6} \log \frac{2}{6}+(2 n-2) \frac{2}{7} \log \frac{2}{7}\right)$

$=\log (1.904761905 n+1.428571429)$

$-\frac{1}{1.904761905 n+1.428571429}(-1.591760035$

$+0.3108960253+0.6361616728+0.3180808364$

$-0.3180808364 n-0.318960253 n-0.3180808364 n$ )

$=\log (1.904761905 n+1.428571429)-\left(\frac{0.1714762878}{n}\right.$

$-0.5014390109-0.2286350503-0.6685853473 n)$

$=\log (1.904761905 n+1.428571429)+0.73007460609$

$-\frac{0.1714762878}{n}+0.6685853473 n$

$=\log (1.904761905 n+1.428571429)+0.73007460609$

$-\frac{0.171476281}{n}+0.6685853473 n$.

Theorem 3.8. For the double row pentachains $G\left(n ; S_{1}\right)$ the weighted entropy with sum connectivity Zagreb index weight is $I(G, S C I)=\log (2.388922108 n+1.266717266)$

$+0.6290155301+\frac{0.12159990486}{n}+0.753781665 n$.

Proof. Using Table 3 values in the definition of sum connectivity index we have $S C I\left(G\left(n, S_{1}\right)\right)=2.388922108 n+1.266717266 I(G, S C I)$ $=\log (S C I)-\frac{1}{S C I} \sum_{g h \in E(G)}\left(\frac{1}{\sqrt{d_{g}+d_{h}}} \log \frac{1}{\sqrt{d_{g}+d_{h}}}\right)$

$=\log (2.388922108 n+1.266717266)$

$-\frac{1}{2.388922108 n+1.266717266}$

(10. $\frac{1}{\sqrt{5}} \log \frac{1}{\sqrt{5}}+(2 n-2) \frac{1}{\sqrt{6}} \log \frac{1}{\sqrt{6}}$

$\left.+(2 n-4) \frac{1}{\sqrt{6}} \log \frac{1}{\sqrt{6}}+(2 n-2) \frac{1}{\sqrt{7}} \log \frac{1}{\sqrt{7}}\right)$ 
$=\log (2.388922108 n+1.266717266)-\left(\frac{0.4185988303}{n}\right.$ $+0.7894421485)(-1.562944444+0.3176789177$ $+0.6353578354+0.3194170353-0.3176789177 n$ $-0.3176789177 n-0.319470353 n)$

$=\log (2.388922108 n+1.266717266)-(-0.1215990486$

$-0.3996899628-0.229355673-0.7537816165 n)$

$=\log (2.388922108 n+1.266717266)+0.6290155301$

$+\frac{0.12159990486}{n}+0.753781665 n$.

\section{Double row pentachains $G\left(n ; S_{2}\right)$}

The order and size of the graph $\mathrm{G}\left(\mathrm{n} ; \mathrm{S}_{2}\right)$ is $5 n+3$ and $7 n+2$ respectively, and the diameter is $n+2, n \geq 2$.

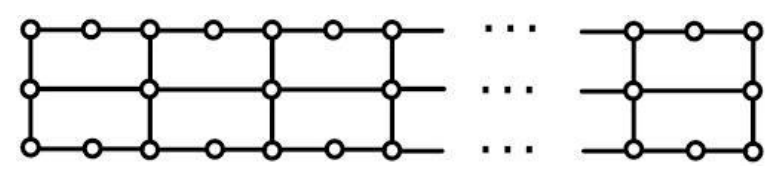

Figure 3 Double row pentachains $\mathrm{G}\left(\mathrm{n} ; \mathrm{S}_{2}\right)$

It can be observed from Figure 3 that there are following four types of edges.

TABLE 4 Edge partition of Double row pentachains $\mathrm{G}\left(\mathrm{n}, \mathrm{S}_{2}\right)$

\begin{tabular}{|c|c|c|c|c|c|}
\hline Edge & $\left(d_{G}(g), d_{G}(h)\right)$ & $(2,2)$ & $(2,3)$ & $(3,4)$ & $(4,4)$ \\
\hline Vertices & Frequencies & 4 & $4 \mathrm{n}$ & $2 \mathrm{n}$ & $\mathrm{n}-2$ \\
\hline
\end{tabular}

Theorem 4.1. For the double row pentachains $G\left(n ; S_{2}\right)$ the weighted entropy with Randić index weight is

$I(G, R)=\log (2.46034343431 n+1.5)+0.6467242417$

$+\frac{0.1223528358}{n}+0.7316037497 n$.

Proof. Using Table 4 values in the definition

of Randić Index we have

$R\left(G\left(n, S_{2}\right)\right)=2.46034343431 n+1.5 I(G, R)$

$=\log (R)-\frac{1}{R} \sum_{g h \in E(G)}\left(\frac{1}{\sqrt{d_{g} \cdot d_{h}}} \log \frac{1}{\sqrt{d_{g} \cdot d_{h}}}\right)$

$=\log (2.46034343431 n+1.5)-\frac{1}{2.46034343431 n+1.5}$

$\left(4 \times \frac{1}{2} \log \frac{1}{2}+4 n \times \frac{1}{\sqrt{6}} \log \frac{1}{\sqrt{6}}\right.$

$\left.+2 \times \frac{1}{\sqrt{12}} \log \frac{1}{\sqrt{12}}+(n-2) \times \frac{1}{4} \log \frac{1}{4}\right)$

$=\log (2.46034343431 n+1.5)-\left(\frac{0.4064473225}{n}\right.$

$+0.6666666667)(-0.6020599913-0.6353578353 n$

$-0.3115327915 n-0.1505149978 n+0.3010299956)$

$=\log (2.46034343431 n+1.5)-\left(-\frac{0.1223528358}{n}\right.$

$-0.4460375778-0.2006866639-0.7316037497 n)$

$=\log (2.46034343431 n+1.5)+0.6467242417$

$+\frac{0.1223528358}{n}+0.7316037497 n$.
Theorem 4.2. For the double row pentachains $\mathrm{G}(\mathrm{n} ; \mathrm{S} 2)$ the weighted entropy with reciprocal Randić index weight is $I(G, R R)=\log (20.7261622014 n)$

$-0.4804932232+\frac{0.1161932413}{n}$.

Proof. Using Table 4 values in the definition of reciprocal Randić index we have $\left.R R\left(G\left(n, S_{2}\right)\right)=20.7261622014 n I(G, R R)\right)$ $=\log (R R)-\frac{1}{R R} \sum_{g h \in E(G)}\left(\sqrt{d_{g} \cdot d_{h}} \log \sqrt{d_{g} \cdot d_{h}}\right)$ $=\log (20.7261622014 n)-\frac{0.0482481991}{n}(4.2 \log 2$ $+4 n \cdot \sqrt{6} \log \sqrt{6}+2 n \cdot \sqrt{12} \log \sqrt{12}+(n-2) \cdot 4 \log 4)$ $=\log (20.7261622014 n)-\frac{0.0482481991}{n}(2.408239965$ $+3.8121470123 n+3.7383934974 n+2.4082399653 n$ $-4.8164799036)=\log (20.7261622014 n)$ $-\left(\frac{0.1161932413}{n}+0.4804932232\right)$

$=\log (20.7261622014 n)-0.4804932232+\frac{0.1161932413}{n}$.

Theorem 4.3. For the double row pentachains $G\left(n ; S_{2}\right)$ the weighted entropy with zagreb Indices weight is
(a) $I\left(G, M_{1}\right)=\log (42 n)-0.7865593459+\frac{0.1146780935}{n}$
(b) $I\left(G, M_{2}\right)=\log (64 n)-0.9975296818+\frac{0.45154375}{n}$. 
Proof. Using Table 4 values in the definition of Zagreb indices we have

(a) $M_{1}\left(G\left(n, S_{2}\right)=42 n I\left(G, M_{1}\right)\right.$

$=\log \left(M_{1}\right)-\frac{1}{M_{1}} \sum_{g h \in E(G)}\left(\left(d_{g}+d_{h}\right) \log \left(d_{g}+d_{h}\right)\right)$

$=\log (42 n)-\frac{1}{42 n} 4.4 \log 4+4 n .5 \log 5+2 n .7 \log 7$

$+(n-2) .8 \log 4=\log (42 n)-\frac{0.0238095238}{n} \times$

$(9.6329598612+13.9794000867 n+11.8313725602 n$

$+7.2247198959 n-14.4494397919)$

$=\log (42 n)-\frac{0.0238095238}{n}(-4.8164799309+33.0354925428 n)$

$=\log (42 n)-0.7865593459+\frac{0.1146780935}{n}$

(b) $M_{2}\left(G,\left(n, S_{2}\right)\right)=64 n I\left(G, M_{2}\right)$

$=\log \left(M_{2}\right)-\frac{1}{M_{2}} \sum_{g h \in E(G)}\left(\left(d_{g} \cdot d_{h}\right) \log \left(d_{g} \cdot d_{h}\right)\right)$

$=\log (64 n)-\frac{0.015625}{n}(4.4 \log 4+4 n .6 \log 6+2 n .12 \log 12$

$+(n-2) 16 \log 16)=\log (64 n)-\frac{0.015625}{n}(9.632959812$

$+18.6756300092 n+25.9003499058 n+19.2659197225 n$

$-38.531839445)=\log (64 n)-\frac{0.015625}{n}(-28.8988795838$

$+63.8418996368 n)=\log (64 n)-0.9975296818+\frac{0.45154375}{n}$.

Theorem 4.4. For double row pentachain $\mathrm{G}\left(\mathrm{n} ; \mathrm{S}_{2}\right)$. The weighted entropy with atombond connectivity index weight is

$I(G, A B C)=\log (4.7317940092 n+1.6036822533)$

$+0.2722078823+\frac{0.0348928507}{n}+0.4998320317 n$.

Proof. Using Table 4 values in the definition of atom-bond connectivity index we have

$A B C(G)=4.7317940092 n+1.6036822533 I(G, A B C)$

$=\log (A B C)-\frac{1}{A B C} \sum_{g h \in E(G)}\left(\sqrt{\frac{d_{g}+d_{h}-2}{d_{g} \cdot d_{h}}} \log \sqrt{\frac{d_{g}+d_{h}-2}{d_{g} \cdot d_{h}}}\right)$

$=\log (4.7317940092 n+1.6036822533)$

$-\frac{1}{4.7317940092 n+1.6036822533}\left(4 . \sqrt{\frac{2+2-2}{2.2}} \log \sqrt{\frac{2+2-2}{2.2}}\right.$

$+4 n \cdot \sqrt{\frac{2+3-2}{2.3}} \log \sqrt{\frac{2+3-2}{2.3}}+2 n \cdot \sqrt{\frac{3+4-2}{3+4}} \log \sqrt{\frac{3+4-2}{3+4}}$

$\left.+(n-2) \cdot \sqrt{\frac{4+4-2}{4+4}} \log \sqrt{\frac{4+4-2}{4+4}}\right)$

$=\log (4.7317940092 n+1.6036822533)-\left(\frac{0.2113363342}{n}\right.$

$+0.62356469225)(-0.4257207025-0.4257207025 n$

$-0.2454253012 n-0.1304257551 n+0.26088515101)$ $=\log (4.7317940092 n+1.6036822533)-\left(-\frac{0.1648691924}{n}\right.$

$-0.169401237-0.10286066452-0.4998320317 n)$

$=\log (4.7317940092 n+1.6036822533)+0.2722078823$

$+\frac{0.0348928507}{n}+0.4998320317 n$.

Theorem 4.5. For double row pentachain $\mathrm{G}\left(\mathrm{n} ; \mathrm{S}_{2}\right)$. The weighted entropy with agumented Zagreb index weight is $I(G, A Z I)=\log (78.61 n-5.92)-4.38822830236$ $+\frac{0.2489110302}{n}+14.3020284944 n$.

Proof. Using Table 4.1 values in the definition of augmented Zagreb index we have $\operatorname{AZI}\left(G\left(n, S_{2}\right)\right)=78.61 n-5.92 I(G, A Z I)$

$=\log (A Z I)-\frac{1}{A Z I} \sum_{g h \in E(G)}\left(\left(\frac{d_{g} \cdot d_{h}}{d_{g}+d_{h}-2}\right)^{3} \log \left(\frac{d_{g} \cdot d_{h}}{d_{g}+d_{h}-2}\right)^{3}\right)$

$=\log (78.61 n-5.92)-\frac{1}{78.61 n-5.92}\left(4 .\left(\frac{2.2}{2+2-2}\right)^{3}\right.$

$\log \left(\frac{2.2}{2+2-2}\right)^{3}+4 n \cdot\left(\frac{2.3}{2+3-2}\right)^{3} \log \left(\frac{2.3}{2+3-2}\right)^{3}+2 n \cdot\left(\frac{3.4}{3+4-2}\right)^{3}$

$\left.\log \left(\frac{3.4}{3+4-2}\right)^{3}+(n-2)\left(\frac{4.4}{4+4-2}\right)^{3} \log \left(\frac{4.4}{4+4-2}\right)^{3}\right)$

$=\log (78.61 n-5.92)-\left(\frac{0.0127210297}{n}-0.1689189189\right)$

$(28.8988795837+28.8988795837 n+31.5362412325 n$

$+24.2328878804 n-48.4657757608)$

$=\log (78.61 n-5.92)-\left(-\frac{0.2489110302}{n}+1.0770641009\right.$

$+3.3052189227-14.3020284944 n)$

$=\log (78.61 n-5.92)-4.38822830236$

$+\frac{0.2489110302}{n}+14.3020284944 n$.

Theorem 4.6. For double row pentachain $G\left(n ; S_{2}\right)$. The weighted entropy with geometric-arithmetic index weight is

$I(G, G A)=\log (6.8986702257 n+2)$

$+0.076053987+0.0262335688 n$.

Proof. Using Table 4 values in the definition of geometric-arithmetic index we have $G A\left(G\left(n, S_{2}\right)\right)=6.8986702257 n+2 I(G, G A)$

$=\log (G A)-\frac{1}{G A} \sum_{g h \in E(G)}\left(\frac{2 \sqrt{d_{g} \cdot g_{h}}}{d_{g}+d_{h}} \log \frac{2 \sqrt{d_{g} \cdot g_{h}}}{d_{g}+d_{h}}\right)$

$=\log (6.8986702257 n+2)-\frac{1}{6.8986702257 n+2}$

(4. $\frac{2 \sqrt{2.2}}{2+2} \log \frac{2 \sqrt{2.2}}{2+2}+4 n \cdot \frac{2 \sqrt{2.3}}{2+3} \log \frac{2 \sqrt{2.3}}{2+3}$

$\left.+2 n \cdot \frac{2 \sqrt{3.4}}{3+4} \log \frac{2 \sqrt{3.4}}{3+4}+(n-2) \frac{2 \sqrt{4.4}}{4+4} \log \frac{2 \sqrt{4.4}}{4+4}\right)$ 
$=\log (6.8986702257 n+2)-\left(\frac{0.1449554722}{n}+0.5\right)$

$(-0.076053987-0.0262335688 n)$

$=\log (6.8986702257 n+2)-(-0.076053987-0.0262335688 n)$

$=\log (6.8986702257 n+2)+0.076053987+0.0262335688 n$.

Theorem 4.7. For double row pentachain $\mathrm{G}(\mathrm{n} ; \mathrm{S} 2)$. The weighted entropy with harmonic index weight is

$I(G, H)=\log (2.42 n-1.5)+0.2530798806$

$+\frac{0.1243925602}{n}-0.7320766914 n$.

Proof. Using Table 4 values in the definition of harmonic index we have

$H I\left(G\left(n, S_{2}\right)\right)=2.42 n-1.5 I(G, H I)$

$=\log (H I)-\frac{1}{H I} \sum_{g h \in E(G)} \frac{2}{d_{g}+d_{h}} \log \frac{2}{d_{g}+d_{h}}$

$=\log (2.42 n-1.5)-\frac{1}{2.42 n-1.5}\left(4 . \frac{2}{2+2} \log \frac{2}{2+2}\right.$

$\left.+4 n \cdot \frac{2}{2+3} \log \frac{2}{2+3}+2 n \cdot \frac{2}{3+4} \log \frac{2}{3+4}+(n-2) \cdot \frac{2}{4+4} \log \frac{2}{4+4}\right)$

$=\log (2.42 n-1.5)-\left(\frac{0.4132231405}{n}-0.666666667\right)$

$(-0.6020599913-0.6367040139 n-0.3108960254 n$

$-0.1505149978 n+0.3010299957)$

$=\log (2.42 n-1.5)-\left(-0.4537665443-\frac{0.1243925602}{n}\right.$

$+0.2006866637+0.752076287 n)=\log (2.42 n-1.5)$

$+0.2530798806+\frac{0.1243925602}{n}-0.7320766914 n$.

Theorem 4.8. For double row pentachain $\mathrm{G}\left(\mathrm{n} ; \mathrm{S}_{2}\right)$. The weighted entropy with sumconnectivity index weight is

$I(G, S C I)=\log (2.8983367186 n+1.2928932188)$

$+1.2350754696+\frac{0.0975626685}{n}+0.8540845133 n$.

Proof. Using Table 4.1 values in the definition of sum connectivity index we have $\operatorname{SCI}\left(G\left(n, S_{2}\right)\right)=2.8983367186 n+1.2928932188 I(G, S C I)$

$=\log (S C I)-\frac{1}{S C I} \sum_{g h \in E(G)}\left(\frac{1}{\sqrt{d_{g}+d_{h}}} \log \frac{1}{\sqrt{d_{g}+d_{h}}}\right)$

$=\log (2.8983367186 n+1.2928932188)-\left(\frac{0.3450254739}{n}\right.$

$+0.7734590802)\left(4 \cdot \frac{1}{\sqrt{2+2}} \log \frac{1}{\sqrt{2+2}}+4 n \cdot \frac{1}{\sqrt{2+3}} \log \frac{1}{\sqrt{2+3}}\right.$

$\left.+2 n \cdot \frac{1}{\sqrt{3+4}} \log \frac{1}{\sqrt{3+4}}+(n-2) \frac{1}{\sqrt{4+4}} \log \frac{1}{\sqrt{4+4}}\right)$

$=\log (2.8983367186 n+1.2928932188)-\left(\frac{0.3450254739}{n}\right.$

$+0.7734590802)(-0.6020599913-0.6251777776 n$

$-0.3194170353 n-0.1596452635 n+0.3192905269)$ $=\log (2.8983367186 n+1.2928932188)-\left(-\frac{0.0975626685}{n}\right.$

$-0.3809909557-0.08540845139-0.8540845133 n)$

$=\log (2.8983367186 n+1.2928932188)+1.2350754696$

$+\frac{0.0975626685}{n}+0.8540845133 n$.

\section{Conclusion}

Numerous procedures for examining the complex networks quantitatively have been contributed. A diversity of problems in discrete mathematics, information theory, statistics, computer science, chemistry and biology are dealing with investigating the entropies for relational structures. In this research study we computed the weighted entropies of the pentagon and double row pentachains by using the probability density function with the help of different topological indices such as Randić index, Zagreb indices, atom-bond connectivity, augmented Zagreb index, geometric arithmetic index, and sum connectivity index as edge weight.

\section{Orcid:}

Deeba Afzal: https://orcid.org/0000-00015268-7260

Farkhanda Afzal: https://orcid.org/00000001-5396-7598

\section{Acknowledgments}

The authors would like to thank the reviewers for their helpful suggestions and comments.

\section{References}

[1] B.Y. Yang, Y.N. Yen, Adv. Appl. Math., 1995, 16, 72-94.

[2] C.E. Shannon, Bell. System Tech. J., 1948, $27,379-423$

[3] C.E. Shannon, W. Weaver, Univ. Illinois Press, Urbana., 1949, 17.

[4] E. Trucco, Bull. Math. Biol., 1965, 18, 129 135. 
[5] K. Xu, K.C. Das, S. Balachandran, $M A T C H$ Commun. Math. Comput. Chem. 2014, 72, 641654.

[6] M.M. Dehmer, N.N. Barbarini, K.K. Varmuza, A.A. Graber, BMC Structural Bio., 2010, 10, 18-34.

[7] N.P. Rao, P.A. Laxmi, Appl. Sci., 2008, 2, 2443-2457.

[8] N. Rashevsky, Bull. Math. Biophys., 1955, 17, 229-235.
[9] S. Ji, X. Li, B. Huo, MATCH Commun. Math. Comput. Chem., 2014, 72, 723-732.

How to cite this article: Farkhanda Afzal, Mehmoona Abdul Razaq, Deeba Afzal*, Saira Hameed. Weighted entropy of penta chains graph. Eurasian Chemical Communications, 2020, 2(6), 652-662. Link: http://www.echemcom.com/article_104806 .html 